\title{
DESIGN, MODELLING AND SIMULATION OF A REMOTELY OPERATED VEHICLE - PART 1
}

\author{
HUNG DUC NGUYEN, SACHITH MALALAGAMA, DEV RANMUTHUGALA \\ University of Tasmania / Australian Maritime College; nguyenhd@amc.edu.au
}

Tóm tắt. Bài báo trình bày mô phỏng số cho một phương tiện ngầm vận hành từ xa (ROV) mới được phát triển gần đây sử dụng lý thuyết và thử nghiệm để thu được đạ̣c tính thủy động lực học và mô hình số dùng LabVIEW để ước lượng động thái của phương tiện ngầm. Nhằm thiết kế và thực hiện điều khiển chính xác một phương tiện ngầm vận hành từ xa trong khi làm nhiệm vụ thì mô hình toán yêu cầu các hệ số thủy động học chính xác được xác định thông qua sự kết hợp giữa phương pháp giải tích, động học chất lỏng tính toán (CFD) và thử nghiệm. Mô phỏng dùng LabVIEW có thể kiểm chứng được các tham số và mô hình toán của phương tiện ngầm vận hành từ xa theo các điều động và điều kiện hoạt động thay đổi.

Từ khóa. Mô hình hóa, $\mathrm{CFD}$, mô phỏng số, điều khiển, phương tiện vận hành từ xa và phương tiện ngầm.

\begin{abstract}
This paper presents the numerical simulation of a recently developed Remotely Operated Vehicle (ROV) utilising theoretical and experimental work to obtain the vehicle's hydrodynamic characteristics and a LabVIEW based numerical model to predict its behaviour. In order to design and to implement precise control of the ROV during missions, the mathematical model requires accurate hydrodynamic coefficients, which are determined for the ROV through a combination of analytical, Computational Fluid Dynamics (CFD), and experimental work. The LabVIEW based simulation enabled the verification of the coefficients and mathematical model under varying operational manoeuvres and conditions.
\end{abstract}

Key words. Modelling, CFD, numerical simulation, control, ROV and underwater vehicle.

\section{Abbreviation}

AMC: Australian Maritime College;

AUV: Autonomous Underwater Vehicle;

CFD: Computational Fluid Dynamics;

CWC: Circulating Water Channel;

DOF: Degrees of Freedom;

GNSS/INS: Global Navigation Satellite System/Inertial Navigation System;

HIL: Hardware in the Loop; PID: Proportional Integral and Derivative;

ROV: Remotely Operated Vehicle;

SST: Shear Stress Transport;

UTAS: University of Tasmania. 


\section{Nomenclature}

\begin{tabular}{|c|c|c|}
\hline Symbol & Unit & Description \\
\hline$B$ & $\mathrm{~N}$ & buoyancy force \\
\hline $\mathbf{C}(\nu)$ & & Coriolis centripetal matrix \\
\hline $\mathbf{D}(\nu)$ & & damping matrix \\
\hline $\mathbf{g}(\eta)$ & & gravitational and buoyancy forces and moments vector \\
\hline$I_{x}, I_{y}, I_{z}$ & $\mathrm{kgm}^{2}$ & Moment of inertia about $x, y$ and $z$ axis, respectively \\
\hline $\mathbf{J}_{\Theta}$ & & Jacobian transform matrix \\
\hline$k$ & $\mathrm{~N} / \mathrm{V}$ & thrust coefficient \\
\hline$K_{P}, K_{I}, K_{D}$ & & PID control gains \\
\hline$l_{i}(i=1,2,3)$ & $\mathrm{m}$ & distance from each thruster to centre of gravity \\
\hline M & & mass matrix \\
\hline $\mathbf{M}_{R B}$ & & rigid body mass matrix \\
\hline $\mathbf{M}_{A}$ & & added mass matrix \\
\hline$p, q, r$ & $\mathrm{rad} / \mathrm{s}$ & roll, pitch and yaw rates \\
\hline $\mathbf{R}_{b}^{n}(\boldsymbol{\Theta})$ & & Euler angle rotation matrix \\
\hline $\mathbf{T}_{\Theta}(\boldsymbol{\Theta})$ & & Euler coordinate system transformation matrix \\
\hline$u$ & & input vector \\
\hline$u, v, w$ & $\mathrm{~m} / \mathrm{s}$ & surge, sway and heave velocities \\
\hline$W$ & $\mathrm{~N}$ & weight \\
\hline$x, y, z$ & $\mathrm{~m}$ & displacements along the $x, y$, and $z$-axes \\
\hline$x_{G}, y_{G}, z_{G}$ & $\mathrm{~m}$ & coordinates of the vehicle's centre of gravity \\
\hline$\eta$ & & $\eta=[x, y, z, \phi, \theta, \psi]^{T}$ the position and Euler's angle vector \\
\hline$\nu$ & & $\nu=[u, v, w, p, q, r]^{T}$ the linear velocity vector \\
\hline$\nu_{r}$ & & $\nu_{r}=\left[u_{r}, v_{r}, w_{r}, p_{r}, q_{r}, r_{r}\right]^{T}$ the relative velocity vector, $\nu_{r}=\nu-\nu_{c}$ \\
\hline$\nu_{c}$ & & current velocity vector \\
\hline$\phi, \theta, \psi$ & $\mathrm{rad}$ & roll, pitch and yaw angles \\
\hline
\end{tabular}

\section{INTRODUCTION}

When designing ROV/AUV platforms requiring precise control during underwater missions $[10,11]$, the physical and virtual/mathematical models play an important role, enabling the designer to understand the vehicles' dynamics and to develop appropriate and adequate control systems. However the development of a specialist physical prototype of a ROV or an AUV, even utilising off-the-shelf electronics is relatively expensive and can significantly limit development, especially within academic institutions developing such vehicles for educational and research purposes. Thus, the authors developed an inexpensive ROV using easily accessible material and equipment [17]. The ROV was fabricated utilising: PVC piping for the vehicle's frame; thrusters developed from submersible bilge pump motors connected to model scaled propellers; fishing net floats for buoyancy; and miscellaneous equipment and components that are easily obtainable from local hardware stores. The ROV was designed to carry out the following [16, 17]:

- observe and survey seabed conditions, submersed objects, and structures;

- observe aquaculture farm facilities and equipment; and

- perform basic underwater surveillance operations. 
Although the vehicle in this paper is tethered, i.e. generally depends on a human operator for guidance and control [21], it can also be untethered with pre-programed mission control, thus operating in AUV mode.

This work further developed the vehicle, control algorithms, mathematical models, and computer simulations to predict the dynamic behaviour of the ROV. Thus, this paper describes the:

- low cost ROV (designated AMC ROV-IV);

- numerical modelling of the ROV/AUV;

- analytical, CFD and experimental work to predict the hydrodynamic coefficients of the ROV;

- simulation of the ROV under various manoeuvring scenarios; and

- design and simulation of a trajectory tracking control system to conduct underwater missions.

\section{DESCRIPTION OF AMC ROV-IV}

The frame of the AMC ROV-IV was fabricated using a combination of PVC pipes and joints, aluminium struts, and lightweight fasteners. The required buoyancy and trim was provided by two longitudinally locatable fishing net floats and adjustable weights as shown in Figs. 1 and 3, with the main particulars of the ROV given in Table 1. Submerged bilge pump motors directly connected to model scale propellers were used for the two propulsion thrusters and the single vertical thruster. All material and components used were obtained through the local hardware and marine suppliers [17]. The ROV was tested for watertight integrity to a depth of 5 metres in the AMC Survival Centre Pool and the Circulating Water Chanel (CWC).

The electrical/electronic equipment consisted of three thrusters, three switch (relay) motor controllers, two forward lights, and relevant instrumentation and control electronics.

Table 1. Main particulars of AMC ROV-IV

$\begin{array}{ll}\text { Length overall }[\mathrm{mm}] & 480 \\ \text { Breadth of vehicle [mm] } & 290 \\ \text { Horizontal distance between } & \\ \text { centres of the two main thrusters [mm] } & 180 \\ \text { Overall width [mm] } & 400 \\ \text { Height without floats [mm] } & 190 \\ \text { Height with floats [mm] } & 225 \\ \text { Weight in air [kg] } & 2.965 \\ \text { Volume }\left[\mathrm{m}^{3} \text { ] }\right. & 2.975 \times 10^{-3}\end{array}$

\section{REFERENCE FRAMES AND EQUATIONS}

\subsection{Reference frames}

In the design of control systems for underwater vehicles, the kinematics and kinetics are described using the reference frames shown in Fig. 2. These include: the Earth-centred reference frames Earth-Centred Earth-Fixed frame (ECEF) $x_{e} y_{e} z_{e}$ and Earth-Centred Inertial Frame (ECIF) $x_{i} y_{i} z_{i}$; and the geographic reference frames North-East-Down (NED) coordinate system $x_{n} y_{n} z_{n}$ and the Body-Fixed Reference Frame (BFRF) $x_{b} y_{b} z_{b}[3,4]$. 


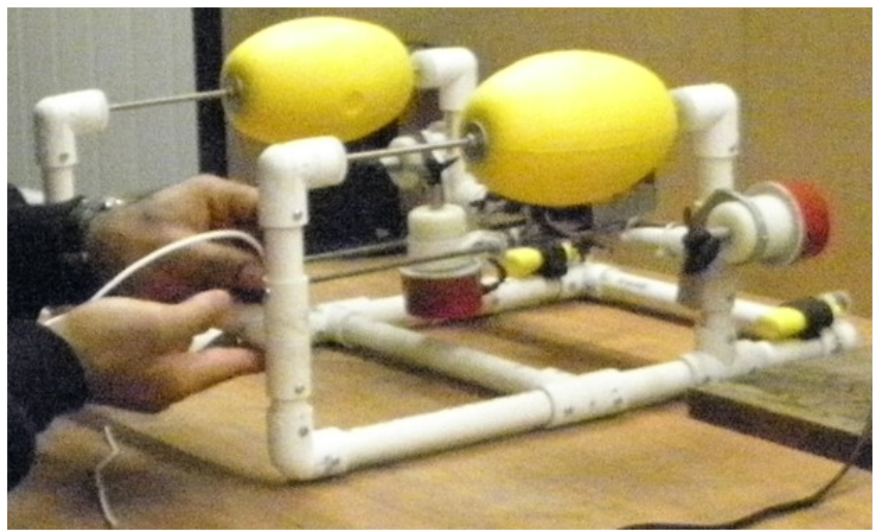

Fig. 1. AMC ROV-IV under construction

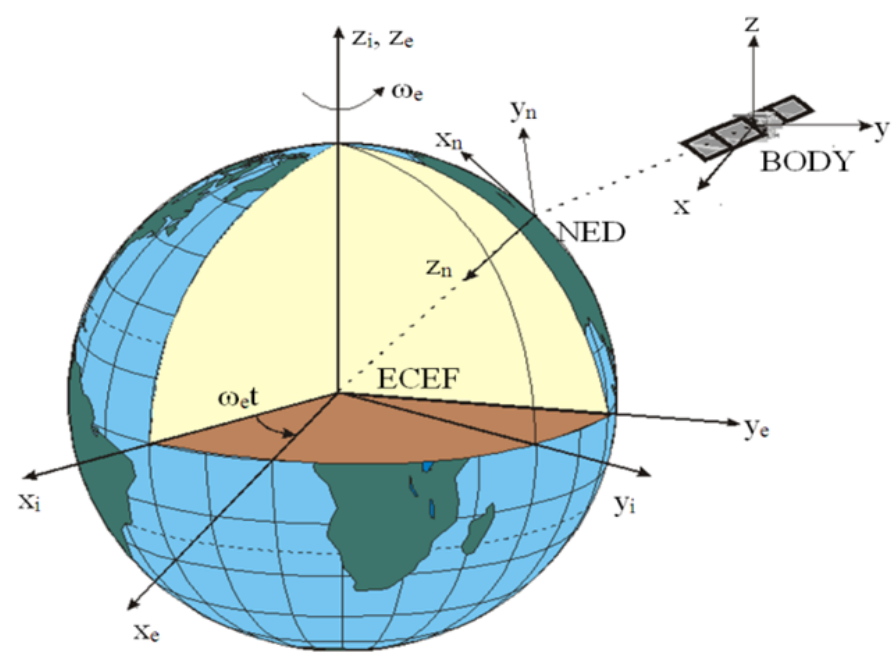

Fig. 2. The Earth-centred and geographic reference frames $[3,4]$

The two reference frames for the AMC ROV-IV are shown in Fig. 3. The NED is the earthfixed reference frame and XYZ is the body-fixed reference frame. The centre of gravity $\mathrm{G}$ is at the vertical central thruster. The arrangement of the three thrusters for position control is shown in Fig. 4. Two floats plus a set of adjustable weights are used to adjust the positions of the centres of buoyancy and gravity and thus the vehicle's trim and heel.

\subsection{Kinematics}

By referring to Fig. 3, according to Fossen $[3,4]$ the 6 -DOF kinematic equations in the Earth-fixed (NED) reference frame in vector form are given by,

$$
\dot{\eta}=\mathbf{J}_{\Theta}(\eta) \nu
$$

where $\eta \in \mathbb{R}^{3} \times S^{3}\left(\mathbb{R}^{3}\right.$ denotes the Euclidean space of dimension three and $S^{3}$ denotes a torus of dimension three, i.e. a sphere) is the position and orientation vector, $\nu \in \mathbb{R}^{3}$ is the linear and angular velocity vector, and $\mathbf{J}_{\Theta}(\eta)$ is expressed by,

$$
\mathbf{J}_{\Theta}(\eta)=\left[\begin{array}{cc}
\mathbf{R}_{b}^{n}(\boldsymbol{\Theta}) & \mathbf{0}_{3 \times 3} \\
\mathbf{0}_{3 \times 3} & \mathbf{T}_{\Theta}(\boldsymbol{\Theta})
\end{array}\right]
$$




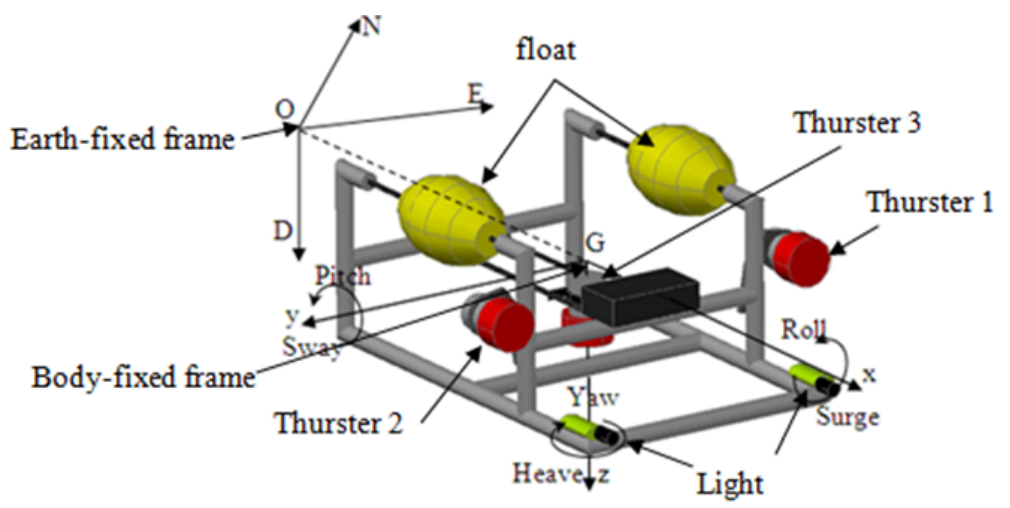

Fig. 3. Reference frames for AMC ROV-IV

where $\mathbf{R}_{b}^{n}(\boldsymbol{\Theta}) \in \mathbb{R}^{3 \times 3}$ is the Euler angle rotation matrix $[3,4]$ between the BODY and NED reference frames and $\mathbf{T}_{\Theta}(\boldsymbol{\Theta})$ is the transformation matrix. Further information on derivation of Equation (1) can be found in [3, 4].

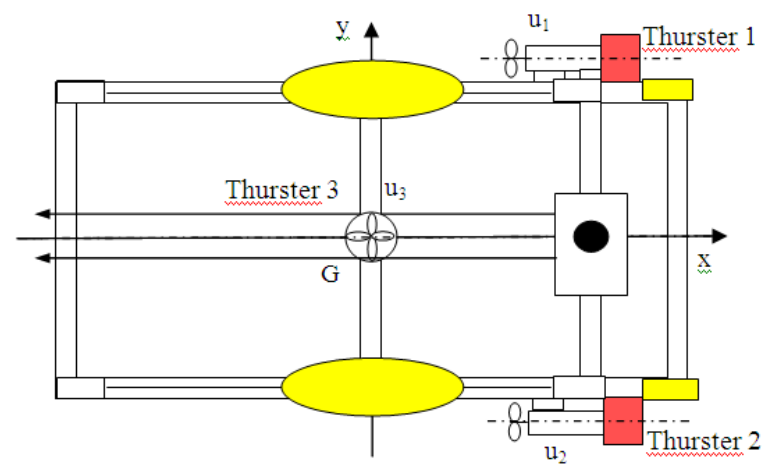

Fig. 4. Arrangement of thrusters of AMC ROV-IV $\left(u_{i}, i=1\right.$ to 3 , voltage inputs of thrusters)

The angle rotation matrix $\mathbf{R}_{b}^{n}(\boldsymbol{\Theta}) \in \mathbb{R}^{3 \times 3}$ is defined in terms of the principal rotations about each axis as $[3,4]$,

$$
\mathbf{R}_{x, \phi}=\left[\begin{array}{ccc}
1 & 0 & 0 \\
0 & c \phi & -s \phi \\
0 & s \phi & c \phi
\end{array}\right] ; \mathbf{R}_{y, \theta}=\left[\begin{array}{ccc}
c \theta & 0 & s \theta \\
0 & 1 & 0 \\
-s \theta & 0 & c \theta
\end{array}\right] \text { and } \mathbf{R}_{z, \psi}=\left[\begin{array}{ccc}
c \psi & -s \psi & 0 \\
s \psi & c \psi & 0 \\
0 & 0 & 1
\end{array}\right]
$$

where $s \cdot=\sin , c \cdot=\cos$. Using the $z y x$ convention we have,

$$
\mathbf{R}_{b}^{n}(\boldsymbol{\Theta}):=\mathbf{R}_{z, \psi} \mathbf{R}_{y, \theta} \mathbf{R}_{x, \phi}
$$

or

$$
\mathbf{R}_{b}^{n}(\boldsymbol{\Theta})=\left[\begin{array}{ccc}
c \psi c \theta & -s \psi c \theta+c \psi s \theta s \phi & s \psi s \phi+c \psi c \phi s \theta \\
s \psi c \theta & c \psi c \phi+s \phi s \theta s \psi & -c \psi s \phi+s \theta s \psi c \phi \\
-s \theta & c \theta s \phi & c \theta c \phi
\end{array}\right]
$$

where the inverse transformation satisfies,

$$
\mathbf{R}_{b}^{n}(\boldsymbol{\Theta})^{-1}=\mathbf{R}_{n}^{b}(\boldsymbol{\Theta})=\mathbf{R}_{x, \phi}^{T} \mathbf{R}_{y, \theta}^{T} \mathbf{R}_{z, \psi}^{T} .
$$


The transformation matrix $\left(\mathbf{T}_{\Theta}(\boldsymbol{\Theta})\right)$ is given by,

$$
\mathbf{T}_{\Theta}(\boldsymbol{\Theta})=\left[\begin{array}{ccc}
1 & s \phi t \theta & c \phi t \theta \\
0 & c \phi & -s \phi \\
0 & s \phi / c \theta & c \phi / c \theta
\end{array}\right] \Rightarrow \mathbf{T}_{\Theta}^{-1}(\boldsymbol{\Theta})=\left[\begin{array}{ccc}
1 & 0 & -s \theta \\
0 & c \phi & c \theta s \phi \\
0 & -s \phi & c \theta c \phi
\end{array}\right] \quad \text { where } \theta \neq \pm 90^{\circ} .
$$

It should be noted that $\mathbf{T}_{\Theta}(\boldsymbol{\Theta})$ is undefined for a pitch angle of $\theta= \pm 90^{\circ}$ and that,

$$
\mathbf{T}_{\Theta}^{-1}(\Theta) \neq \mathbf{T}_{\Theta}^{T}(\Theta) .
$$

\subsection{Kinetic model with disturbances}

Based on the Newton-Euler formation for the rigid bodies and the foundation of vectorial mechanics, the 6-DOF nonlinear dynamic equations of motion in the body-fixed reference frame (as shown in Fig. 3) in vector form are [3, 4],

$$
\mathbf{M} \dot{\nu}+\mathbf{C}(\nu) \nu+\mathbf{D}(\nu) \nu+\mathbf{g}(\eta)+\mathbf{g}_{0}=\tau+\tau_{\text {wind }}+\tau_{\text {wave }}
$$

where $\mathbf{M}$ : system inertia matrix (including the added mass);

$\mathbf{C}(\nu)$ : coriolis-centripetal matrix (including the added mass);

$\mathbf{D}(\nu)$ : damping matrix;

$\mathbf{g}(\eta)$ : gravitational and buoyancy forces and moments vector;

$\mathrm{g}_{0}$ : pre-trimming (ballast control) vector;

$\tau$ : control input vector;

$\tau_{\text {wind }}$ : wind-induced forces and moments vector; and

$\tau_{\text {wave }}$ : wave-induced forces and moments vector.

In order to improve the performance of the ROV, it is necessary to consider the external disturbances on the vehicle when designing its control system, which include the effects of wind, waves, and currents on it. According to Fossen [3, 4], when designing control systems it is common to assume the principle of superposition with regard to current, wind and wave disturbances. In general, the environmental forces and moments will be highly nonlinear and both additive and multiplicative to the dynamic equations of motion. Therefore, an accurate description of the environmental forces and moments is important in underwater vehicle simulators and provides useful information to the human operator. According to Fossen [4], in the case of irrotational ocean current, the relative velocity vector $\nu_{r}=\nu-\nu_{c}$, $\nu_{c}=\left[\begin{array}{llllll}u_{c} & v_{c} & w_{c} & 0 & 0 & 0\end{array}\right]^{T}$ contributes to the hydrodynamic terms such that,

$$
\underbrace{\mathbf{M}_{R B} \dot{\nu}+\mathbf{C}_{R B}(\nu) \nu}_{\text {rigid-body forces }}+\underbrace{\mathbf{M}_{A} \dot{\nu}_{r}+\mathbf{C}_{A}\left(\nu_{r}\right) \nu_{r}+\mathbf{D}\left(\nu_{r}\right) \nu_{r}}_{\text {hydrodynmic forces }}+\underbrace{\mathbf{g}(\eta)+\mathbf{g}_{0}}_{\text {hydrostatic forces }}=\tau+\tau_{\text {wind }}+\tau_{\text {wave }} .
$$

The model in Equation (9) can be rewritten in the following form using the relative velocity vector,

$$
\mathbf{M} \dot{\nu}_{r}+\mathbf{C}\left(\nu_{r}\right) \nu_{r}+\mathbf{D}\left(\nu_{r}\right) \nu_{r}+\mathbf{g}(\eta)+\mathbf{g}_{0}=\tau+\tau_{\text {wind }}+\tau_{\text {wave }}
$$

where $\mathbf{M}=\mathbf{M}_{R B}+\mathbf{M}_{A}\left(\mathbf{M}_{R B}\right.$ is rigid-body mass matrix and $\mathbf{M}_{A}$ is added mass matrix) and $\mathbf{C}\left(\nu_{r}\right)=\mathbf{C}_{R B}\left(\nu_{r}\right)+\mathbf{C}_{A}\left(\nu_{r}\right)\left(\mathbf{C}_{R B}\left(\nu_{r}\right)\right.$ is the rigid body Coriolis-centripetal matrix and $\mathbf{C}_{A}$ the added Coriolis-centripetal matrix). Further information on modelling environmental disturbances can be found in $[2,3,4]$. 


\subsection{Mathematical model for the AMC ROV without disturbances}

In order to derive the differential equations governing the kinematics and dynamics of the vehicle, with the inputs and outputs as shown Fig. 5, it is assumed that:

- the origin of the body-fixed reference frame is at the centre of gravity of the vehicle, which coincides with the location of the vertical thruster;

- the vehicle is symmetric about the longitudinal axis $x$;

- the body has an equivalent block shape, i.e. the body looks like a block with a large block coefficient;

- the vehicle is neutrally buoyant and the mass distribution of the vehicle is homogeneous throughout the vehicle; and

- no external disturbances for simplicity.

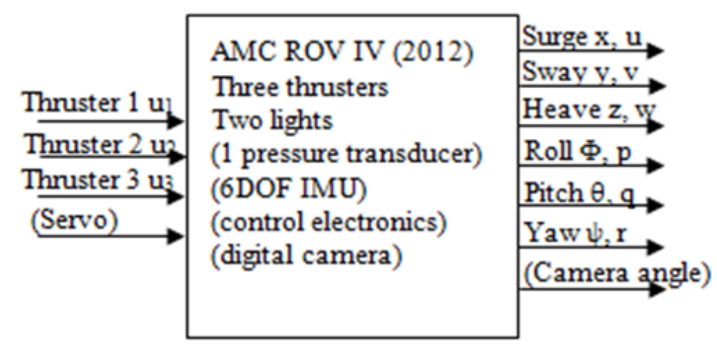

Fig. 5. Input and output variables of the AMC ROV/AUV-IV

Thus, the 6-DOF model in Equation (9) is applied to the AMC ROV-IV as follows as in $[2,3,4,10]$.

The set of equations for the ROV's kinematics are,

$$
\dot{\eta}=\mathbf{J}(\eta) \nu
$$

while the equations for the ROV's kinetics without external disturbances are given by,

$$
\mathbf{M} \dot{\nu}+\mathbf{C}(\nu) \nu+\mathbf{D}(\nu) \nu+\mathbf{g}=\mathbf{B u}
$$

where $\nu=\left[\begin{array}{llllll}u & v & w & p & q & r\end{array}\right]^{T}, \mathbf{J}_{\Theta}(\eta)$ as defined in Equation $(2), \mathbf{B u}=\tau$,

$$
\begin{aligned}
\mathbf{M} & =-\left[\begin{array}{cccccc}
m-X_{\dot{u}} & 0 & 0 & 0 & 0 & 0 \\
0 & m-Y_{\dot{v}} & 0 & 0 & 0 & 0 \\
0 & 0 & m-Z_{\dot{w}} & 0 & 0 & 0 \\
0 & 0 & 0 & I_{x}-K_{\dot{p}} & 0 & 0 \\
0 & 0 & 0 & 0 & I_{y}-M_{\dot{q}} & 0 \\
0 & 0 & 0 & 0 & 0 & I_{z}-N_{\dot{r}}
\end{array}\right], \\
\mathbf{C}(\nu)= & {\left[\begin{array}{cccccc}
0 & -m r & m q & 0 & -Z_{\dot{w}} w & Y_{\dot{v}} v \\
m r & 0 & -m p & Z_{\dot{w}} w & 0 & -X_{\dot{u}} u \\
-m q & m p & 0 & -Y_{\dot{v}} v & X_{\dot{u}} u & 0 \\
0 & -Z_{\dot{w}} w & Y_{\dot{v}} v & 0 & \left(I_{z}-N_{\dot{r}}\right) r & -\left(I_{y}-M_{\dot{q}}\right) q \\
Z_{\dot{w}} w & 0 & -X_{\dot{u} u} u & -\left(I_{z}-N_{\dot{r}}\right) r & 0 & \left(I_{x}-K_{\dot{p}}\right) p \\
-Y_{\dot{v}} v & X_{\dot{u}} u & 0 & -\left(I_{y}-M_{\dot{q}}\right) q & -\left(I_{x}-K_{\dot{p}}\right) p & 0
\end{array}\right], }
\end{aligned}
$$




$$
\begin{gathered}
\mathbf{D}(\nu)=\operatorname{diag}\left\{X_{u}+X_{u|u|}|u|, \quad Y_{v}+Y_{v|v|}|v|, \quad Z_{w}+Z_{w|w|}|w|\right. \\
\left.K_{p}+K_{p|p|}|p|, \quad M_{q}+M_{q|q|}|q|, \quad K_{r}+K_{r|r|}|r|\right\} \\
\mathbf{g}(\eta)=\left[\begin{array}{c}
k \\
0 \\
0 \\
z_{B} B \cos \theta \sin \phi \\
z_{B} B \sin \theta \\
0
\end{array}\right], \mathbf{B}=\left[\begin{array}{ccc}
k & 0 \\
0 & 0 & k \\
0 & 0 & 0 \\
k l_{1} & k l_{2} & -k l_{3} \\
k l_{3} & k l_{3} & 0
\end{array}\right], \quad \text { and } \mathbf{u}=\left[\begin{array}{c}
u_{1} \\
u_{2} \\
u_{3}
\end{array}\right] .
\end{gathered}
$$

The determination of the coefficients in equation (12) is discussed in the following sections.

\section{PARAMETER IDENTIFICATION USING CFD METHOD}

The performance of the ROV without the effects of the external disturbances is described by equations given in equation (12). It is therefore essential to estimate with relative accuracy all of the coefficients in the system of equations given by equation (12), which in this case was carried out using a combination of theoretical work, CFD modelling, and captive experimental techniques. The parameters to be estimated include the added mass, added moments of inertia, thruster coefficients, and damping derivatives.

The translational added mass due to the translational accelerations of the vehicle were estimated by analytical methods using the vehicle's geometrical parameters. This consisted of calculating the added mass for each component of the ROV for each of the three translational motions, i.e. surge, sway and heave, and then arithmetically adding them to obtain the complete added mass of the ROV in that direction $[19,20]$. Where the added mass for required shapes in two dimensional potential flows were not available, the projected area of the component for the given direction was obtained and using its principle dimensions the added mass in that direction was calculated using the following formula [19, 20].

$$
M_{i i}=\frac{4}{3} \frac{\pi \rho\left(a_{i}^{2}\right)\left(b_{i}^{2}\right)}{\left(a_{i}+b_{i}\right)}
$$

where $M_{i i}$ is the translational added mass, and $a_{i}$ and $b_{i}$ are two principal dimensions of the projected area (with $b_{i}>a_{i}$ ). The added moments of inertia around each axis of rotation was estimated by assuming it to be half of the moment of inertia around that particular axis [17].

The thruster coefficients were obtained through experimental means using two spring scales attached to the thruster via a clamp. The thruster force was recorded at different motor voltages, with the coefficient determined by the slope of the graph between the two parameters [17].

CFD simulations of the ROV (Fig. 6) were used to determine the linear and quadratic damping derivatives, with the results supplemented and validated against the captive model experiments of the ROV in AMC's CWC (Fig. 7) [17].

The CFD was carried out using the commercial software ANSYS-CFX, with the CFD modelling carried out in 3D using ANSYS Design Modeller and Mesh Application. A grid independence study was conducted to ensure a grid sensitivity of less than $5 \%$, with the model settings and boundary/initial conditions set to provide steady-state simulations using a Shear 


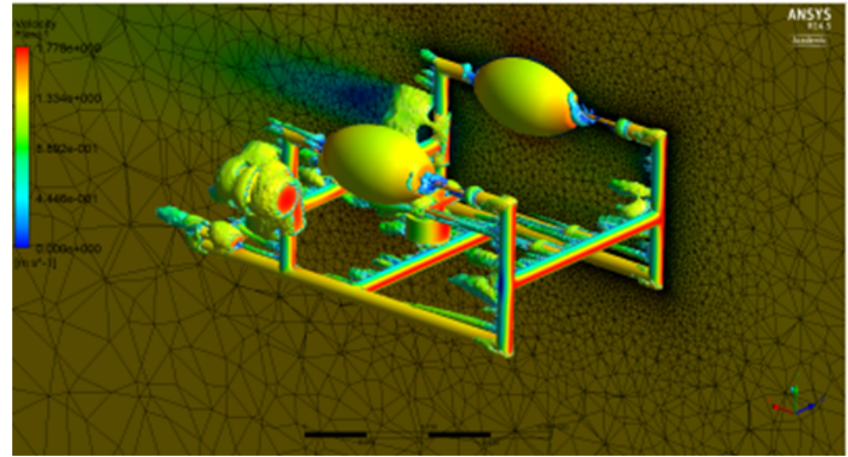

Fig. 6. CFD mesh and pressure contours of the ROV

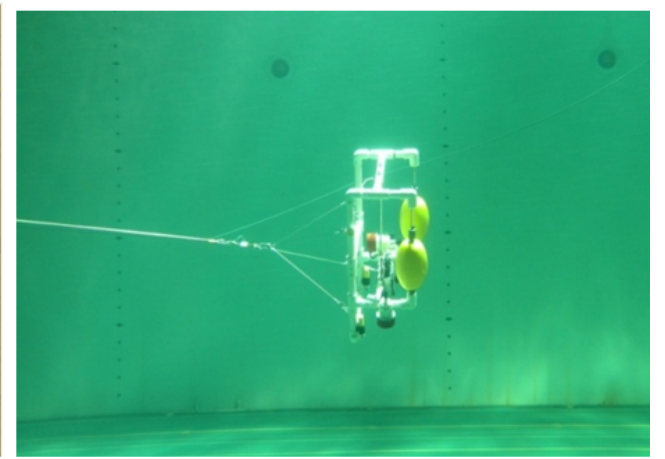

Fig. 7. Drag test of ROV in the CWC

Stress Transport (SST) turbulence model. The CFD simulations were run for translational and rotational motion to estimate the lift and drag forces and the relevant moments at the typical operational speeds. The coefficients were then calculated by non-dimensionalising the forces and moments based on the velocity, geometric parameters (e.g. area), and fluid density. All coefficients for Equation (12) are given in Table 2. Detailed information on the CFD modelling techniques, experiments, and coefficients is given by the authors in [17].

Table 2. Estimated coefficients for Equations (11) and (12)

\begin{tabular}{|l|l|l|l|l|l|l|l|}
\hline Coef & Value & Coef & Value & Coef & Value & Coef & Value \\
\hline$L$ & $480 \mathrm{~mm}$ & $\mathrm{~b}$ & $290 \mathrm{~mm}$ & $Y_{\dot{v}}$ & $-1.919 \mathrm{~kg}$ & $Y_{v|v|}$ & $-16.22 \mathrm{kgm}^{-1}$ \\
\hline$m$ & $2.965 \mathrm{~kg}$ & $I_{x}$ & $0.067 \mathrm{kgm}^{2}$ & $Z_{\dot{w}}$ & $-2.11 \mathrm{~kg}$ & $Z_{w|w|}$ & $-18.8 \mathrm{kgm}^{-1}$ \\
\hline$I_{y}$ & $0.091 \mathrm{kgm}^{2}$ & $I_{z}$ & $0.05 \mathrm{kgm}^{2}$ & $K_{\dot{p}}$ & $-0.0335 \mathrm{kgm}^{2}$ & $K_{p|p|}$ & $-0.07 \mathrm{kgm}$ \\
\hline$B$ & $29.08 \mathrm{~N}$ & $l_{1}$ & 0 & $M_{\dot{q}}$ & $-0.045 \mathrm{kgm}^{2}$ & $M_{q|q|}$ & $-0.17 \mathrm{kgm}$ \\
\hline$l_{2}$ & 0 & $l_{3}$ & $0.18 \mathrm{~m}$ & $N_{\dot{r}}$ & $-0.025 \mathrm{kgm}^{2}$ & $N_{r|r|}$ & $-0.11 \mathrm{kgm}$ \\
\hline$x_{b}$ & 0 & $y_{b}$ & 0 & $X_{u}$ & $-0.59 \mathrm{kgs}^{-1}$ & $K_{p}$ & $-0.02 \mathrm{kgms}^{-1}$ \\
\hline$z_{b}$ & 0 & $\mathrm{~K}$ & $0.373 \mathrm{Nm} / \mathrm{V}$ & $Y_{v}$ & $-0.66 \mathrm{kgs}^{-1}$ & $M_{q}$ & $-0.06 \mathrm{kgms}^{-1}$ \\
\hline$X_{\dot{u}}$ & $-1.251 \mathrm{~kg}$ & $X_{u|u|}$ & $-11.25 \mathrm{kgm}^{-1}$ & $Z_{w}$ & $-0.65 \mathrm{kgs}^{-1}$ & $N_{r}$ & $-0.04 \mathrm{kgms}^{-1}$ \\
\hline
\end{tabular}

Hydrodynamic coefficients in Table 2 are defined as follows:

- $X_{\dot{u}}, Y_{\dot{v}} . Z_{\dot{w}}, K_{\dot{p}}, M_{\dot{q}}, N_{\dot{r}}$ are added mass and moment of inertia coefficients;

- $X_{u}, Y_{v}, Z_{w}, K_{p}, M_{q}, N_{r}$ are linear damping coefficients; and

- $X_{u|u|}, Y_{v|v|}, Z_{w|w|}, K_{p|p|}, M_{q|q|}, K_{r|r|}$ are quadratic damping coefficients.

\section{SIMULATION STUDY}

\subsection{Control design}

The automatic control system for the ROV as a whole is illustrated in Fig. 8 showing the signal flow for the guidance, navigation, and control systems, which are explained below.

Guidance system: to receive prior information, predefined inputs, waypoints, and generate desired trajectory including desired speed, depth (heave), yaw, and position. A joystick may be used to generate reference signals $[3,4,7]$. 


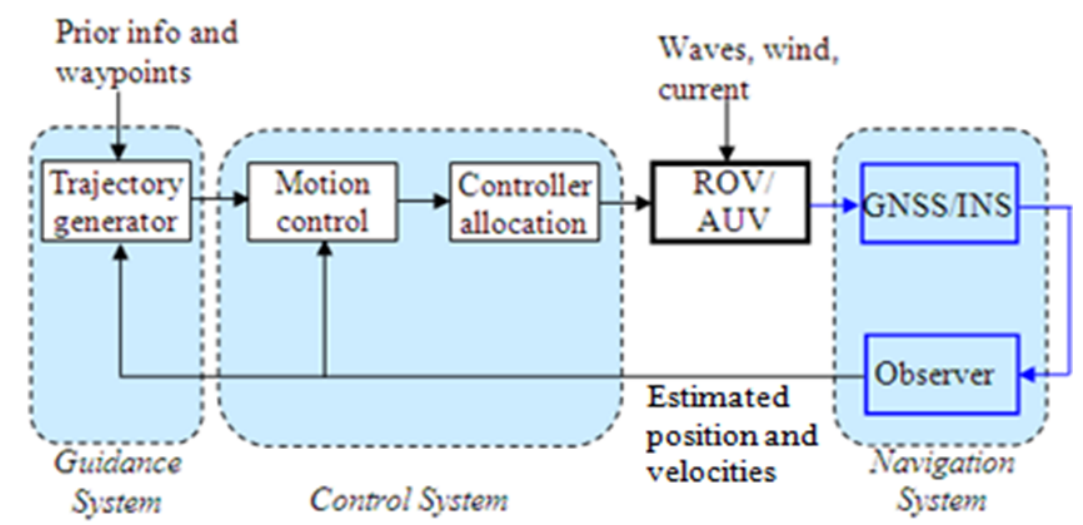

Fig. 8. Guidance, navigation and control systems

Navigation system: equipped with GNSS/INS receivers and other sensors to provide measurement of speed, depth, yaw, and position [3, 4, 7].

Control system: to detect the respective errors by comparing actual speed, depth, heading angle, and position with the desired values; and calculating the control signals to be sent to the controller allocation devices (actuators) $[3,4,7]$. The control system parameters need to be tuned to ensure the vehicle follows the desired inputs with minimum deviations. The simulation results will enable the parameters to be optimised.

As the first step to realize a hardware-in-the-loop system, computer simulation programs were developed in LabVIEW using the mathematical model described in Equations (11) and (12). A number of tests were carried out for the simulation programs, including open-loop system manoeuvres and closed-loop control system tests. The open-loop system manoeuvres were turning circle, horizontal zigzag, and depth zigzag tests. The closed-loop control system tests were course control (course keeping and changing); speed, depth (heave) and pitch control; roll, surge and sway control; and trajectory tracking control.

In order to verify the mathematical model, a trajectory tracking control system was designed. In the simulation programs for closed-loop control systems (including depth and course keeping, pitch and roll control, and position/trajectory tracking control) the conventional PID control law was used due to its simplicity, i.e.,

$$
u=K_{P} e(t)+K_{I} \int e(t) d t+K_{D} \frac{d e(t)}{d t}
$$

where $u$ is the control signal, $e(t)$ is the error, and $K_{P}, K_{I}$ and $K_{D}$ are proportional, integral and derivative control gains, respectively.

\subsection{Open-loop system manoeuvres}

Numerical simulations for open-loop system manoeuvres were carried out using Equations (11) and (12) with the estimated hydrodynamic coefficients in Table 2. They investigated the ROV's behaviour without any feedback signals, with the ROV's thrusters controlled by three relay-based motor drives. The simulated manoeuvres presented here include driving, course changing, course keeping, and circular diving. Open-loop system manoeuvres were carried out, including horizontal zigzag, horizontal turning circle, and depth zigzag manoeuvres. An important test was the circular diving manoeuvre with the vehicle's trajectory shown in Fig. 9 where the vehicle dives along the desired spiral trajectory. 


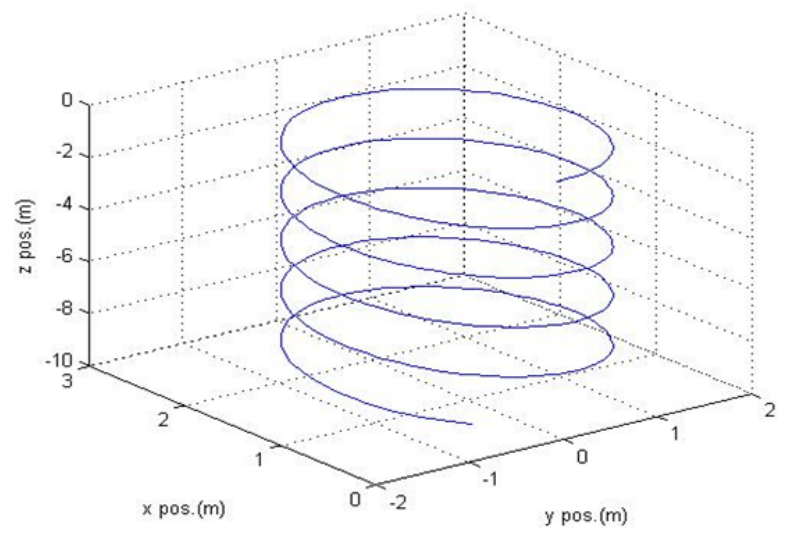

Fig. 9. Open-loop trajectory of circular diving manoeuvre

\subsection{Trajectory tracking control manoeuvres}

A ROV/AUV often does various underwater missions that are based on predefined trajectories, for example, water sampling, survey of submerged pipelines and cables, or hydrographical surveys. Therefore, it is essential to have a good understanding of the vehicle's behaviour during these manoeuvres. Thus, in order to quantify the behaviour of the modelled ROV, a series of closed-loop control manoeuvring simulations were carried out, which included a trajectory tracking control for an underwater mission.

In this manoeuvre, the trajectory tracking control algorithm utilised the way-points tracking Line of Sight (LOS) method. A set of waypoints $\left(x_{k}, y_{k}, z_{k}\right.$, where $\left.k=1,2,3, \ldots, N\right)$ were supplied as inputs resulting in the generation of a desired trajectory, including the desired course $\left(\psi_{d}\right)$, speed $\left(u_{d}\right)$, and position $\left(x_{d}, y_{d}, z_{d}\right)$. The desired course was obtained by the following relationship $[4,5,7,9]$,

$$
\psi_{d}(k)=\tan ^{-1}\left(\frac{y_{k}-y(t)}{x_{k}-y(t)}\right) .
$$

The new course, depth, and speed were selected by the circle of acceptance method $[4,5,7$, 9] as follows,

$$
\left[x_{k}-x(t)\right]^{2}+\left[y_{k}-y(t)\right]^{2}+\left[z_{k}-z(t)\right]^{2} \leqslant R_{0}^{2}
$$

where the coordinate $(x, y, z)$ is the current position and $R_{0}$ is often chosen as twice the vehicle's length $(2 \mathrm{~L})[4,5]$. The desired trajectory is generated based on a simulation program using a simplified mathematical model of the ROV [4, 7].

Fig. 10 shows simulated results of the tracking control for a desired 3D trajectory for which the waypoints were defined as

$$
\begin{aligned}
& X_{w p}=[0,10,10,0,0,0,0,-10,-10,0,0], \\
& Y_{w p}=[0,0,10,10,0,0,-10,-10,0,0,0], \text { and } \\
& Z_{w p}=[0,0,0,0,-10,-10,-10,-10,-10,0] .
\end{aligned}
$$

It is seen from Fig. 10 that the controlled ROV followed the pre-defined 3D trajectory accurately, with Fig. 11 showing the time histories of the linear and angular rates for the vehicle. From Figs. 10 and 11 it is seen that the overshoots for system responses are relatively small or zero, a testimony to the selected control gains that were tuned using the simulation results. 


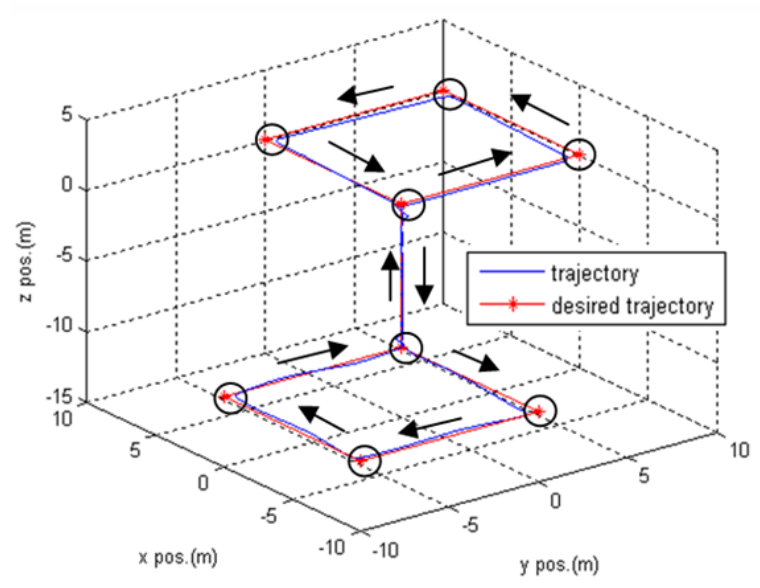

Fig. 10. ROV trajectory for underwater mission
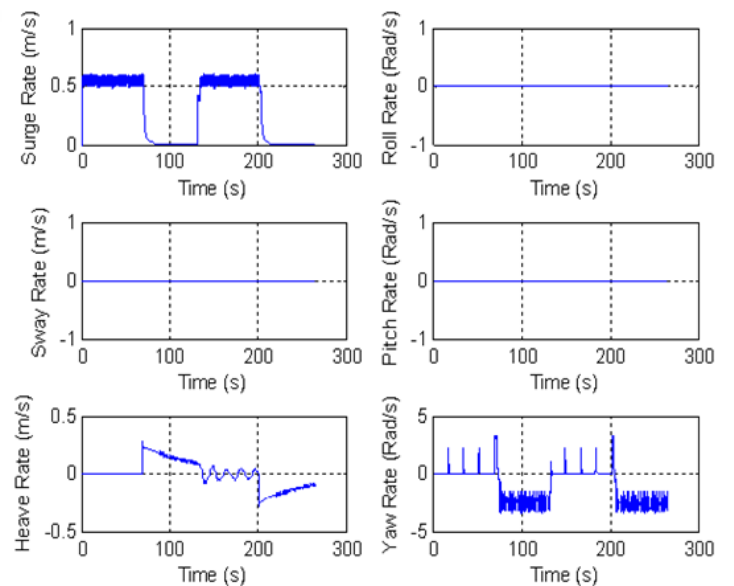

Fig. 11. ROV time history of rates

The accuracy of determining the position of a ROV/AUV depends on the method used. There are several methods to determine an underwater vehicle's position. To enhance accuracy of underwater positioning for a ROV/AUV it is proposed that an underwater acoustic positioning method as in [22] is used in combination with GPS buoys, a Kalman or an extended Kalman filter and an optimal/adaptive observer. See $[10,22,23]$ for more detail on underwater acoustic positioning methods.

\section{CONCLUSIONS}

This paper described the design, development, and construction of an inexpensive ROV using easily accessible materials; and the numerical modelling and simulation of the vehicle. The latter included the development of a 6-DOF mathematical model, with the hydrodynamic coefficients determined through a combination of theoretical, CFD, and experimental work. Numerical simulation studies using LabVIEW software were carried out to verify the developed mathematical models. The simulation study consisted of a number of manoeuvring scenarios under varying operational conditions, including open-loop manoeuvres and closed-loop control manoeuvres with PID control law. The numerical simulation results showed the feasibility of the developed control algorithms for various manoeuvres of the ROVs. The simulated results also showed that the mathematical model with the estimated hydrodynamic coefficients is sufficiently accurate to describe the performance and behaviour of the ROV. It is essential that the coefficients are accurately calculated for the varying conditions, thus enabling the simulation results to be used to optimise the control algorithm.

In order to further understand the dynamic characteristics of the ROV, to upgrade it to an AUV and to design effective controllers for various underwater missions, the following work will be carried out. Installation of control electronics and the development of high-performance control programs using advanced or intelligent control algorithms within MATLAB/Similink or Arduino software. The numerical model and simulations will need validation against data from manoeuvring tests of the physical ROV conducted within a controlled experimental environment. The trajectory tracking control system for underwater missions will be verified by experiments using an underwater acoustic positioning system. Effects of external disturbances such as waves and current on the trajectory and system responses will be considered. These updates will be reported in Part 2 of this paper. 


\section{REFERENCES}

[1] G.N. Roberts, and R. Sutton, (Editors). Advances in Unmanned Marine Vehicles. The Institute of Electrical Engineers, 2006.

[2] T.I. Fossen, "Nonlinear Modelling and Control of Underwater Vehicles", PhD Thesis, Norwegian Institute of Technology, 1991.

[3] T.I. Fossen, Handbook of Marine Craft Hydrodynamics and Motion Control, John Wiley and Sons Inc. 2011.

[4] T.I. Fossen, Marine Control Systems - Guidance, Navigation and Control of Ships, Rigs and Underwater Vehicles. Marine Cybernetics, Trondheim, Norway, 2002.

[5] T.I. Fossen, Guidance and Control of Ocean Vehicles, John Wiley and Sons, 1994.

[6] S.A. Wadoo, and P. Kachoroo, Autonomous Underwater Vehicles: Modeling, Control Design, and Simulation, CRC Press, 2011.

[7] H.D. Nguyen, Multitask manoeuvring systems using recursive optimal control algorithms, Proceedings of HUT-ICCE, Hoi An, Vietnam, 2008 (54-59).

[8] H.D. Nguyen, Recursive identification of ship manoeuvring dynamics and hydrodynamics, ANZIAM Journal 49 (2) (2008) 717-732 (ISSN: 1446-8735).

[9] H.D. Nguyen, Recursive optimal manoeuvring systems for maritime search and rescue mission, Proceedings of OCEANS'04 MTS/IEEE/TECHNO-OCEAN'O4 (OTO'O4), Kobe, Japan, 2004 (911-918).

[10] H.D. Nguyen, R. Pienaar, D. Ranmuthugala, and W. West, Modelling, simulation and control of underwater vehicles, Proceedings of The 1st Vietnam Conference on Control and Automation, Hanoi, Vietnam, 25-26 November 2011 (150-159).

[11] W.J. West, "Remotely Operated Underwater Vehicle", BE Thesis. Australian Maritime College, UTAS, Launceston, 2009.

[12] C.R. Gaskin, "Design and Development of ROV/AUV", BE Thesis. Australian Maritime College, UTAS, Launceston, 2000.

[13] G. Antonelli, Underwater Robots - Motion and Force Control of Vehicle-Manipulated Systems (2nd Edition) Springer, 2006.

[14] R. Burcher, and L. Rydill, Concepts in Submarine Design, Cambridge University Press, 1994.

[15] R.D. Christ, and R.L. Wernli Sr, The ROV Manual - A User Guide for Observation Class Remotely Operated Vehicles, Butter-Heinemann, Elsevier (Oxford, 1994), 2007.

[16] R. Pienaar, "Simulation and Modelling of ROVs and AUVs," BE Thesis. Australian Maritime College, Launceston, 2011.

[17] S. Malalagama, "Modelling and Simulation of a Remotely Operated Vehicle", BE Thesis. Australian Maritime College, University of Tasmania, Launceston. 2012.

[18] H. Blohm, \& V. Jensen, Build Your Own Underwater Robot and Other Wet Projects, Vancouver, Westcoast Words, 1997.

[19] C. E. Brennen, "A Review of Added Mass and Fluid Inertial Forces. Department of the Navy", Port Hueneme, CA, USA. 1982 (Accessed on 4/7/2013 at http://authors.library.caltech.edu/233/).

[20] Y. H. Eng, W. S. Lau, E. Low, G. G. L. Seet, and C. S. Chin, Estimation of the hydrodynamics coefficients of an ROV using free decay pendulum motion, Engineering Letters 16 (3) (2008).

[21] T. I. Fossen, "Non Linear Modelling and Control of Underwater Vehicles," Norwegian Institute of Technology, Trondheim. 1987. 
[22] IMCA (2009). Deep Water Acoustic Positioning. Accessed on 1/9/2013 at http://www.imcaint.com/media/73578/imcam200.pdf

[23] J.C. Kinsey, R.M. Eustice, and L.L. Whitcomb, A survey of underwater vehicle navigation: recent advances and new challenges, Proceedings of MCMC 2006, Lisbon, Portugal, September 2006 (Accessed on 1/9/2013 at http://www.whoi.edu/cms/files/mcmc2006_41265.pdf).

Received on July 08, 2013

Revised on October 16, 2013 